\title{
ON VON BABO'S LAW AND KIRCHHOFF'S EQUATION FOR THE LATENT HEAT OF DILUTION
}

\author{
By ALFRED W. PORTER, D.Sc., F..R.S
}

\author{
(A Paper read before the Faraday Socicty on Tuesday, May II, I9I5, \\ Sir Robert Hadfield, F.R.S., President, in the Chair.)
}

In deriving von Babo's law connecting the vapour pressure $\pi$ of a dilute solution with the vapour pressure $\pi_{\circ}$ of the pure solvent at the same temperature, it is customary to make approximations throughout the course of the investigation which leave one at the end in a state of uncertainty as to the degree of accuracy of the final result. It seems desirable, therefore, to proceed as far as possible without making any approximation, even though the exact equations thus obtained are much more than sufficient in the present state of our experimental knowledge of ordinary solutions. These expressions, moreover, become necessary as the critical point is approached, for the usual approximations cannot then be considered good enough. There is therefore need that they should be developed in order to prepare the way for these more complicated cases.

The following notation will be employed :-

$\mathrm{H}$, the latent heat of reversible dilution, i.e. the heat that must be taken in to keep the solution at constant temperature $T$ and pressure $p$ when I gram of the solvent at the same temperature and at the pressure $p_{0}$ is added through a semi-permeable membrane to a large quantity of it.

$\mathrm{H}_{i}$, the internal latent heat of dilution, i.e. the increment of specific energy of the system when the solution is diluted as above. In the case of incompressible solutions this will not differ appreciably from the irreversible latent heal of dilution or heat absorbed when I gram of solvent is simply stirred into a solution, both being at the same temperature and pressure. This similarity in value is due to the very small amount of external work done by the system during such mixture.

$\pi_{0}, \pi_{\pi}$, the principal saturation pressures of solvent and solution respectively, i.e. the values when the liquids are under the pressures of their vapours alone.

$u_{p_{0}}$, specific volume of the solvent at pressure $p_{0}$.

$s_{p}$, increase of volume of solution at pressure $p$ when I gram of solvent is added to a large quantity of it. The suffixes may be dropped whenever the meaning is otherwise clear.

$\mathrm{P}$, osmotic pressure $=p-p_{0}$.

Special terms are defined when they occur.

The following general theorems may be recalled: Heat that enters unit 


\section{ON VON BABO'S LAW AND KIRCHHOFF'S EQUATION}

mass of fluid (liquid or gas) when its pressure is increased at constant temperature is-

$$
-\mathrm{T}\left(\frac{\partial v}{\partial \mathrm{T}}\right)_{p} d p
$$

where $v$ is the specific volume. The corresponding increase in the specific energy is obtained by subtracting from the above the external work $p d v$ (or $d(p v)$ - vdp), leaving-

$$
-\left(\mathrm{T} \frac{\partial v}{\partial \mathbf{T}}-v\right) d p-d(p v)
$$

or-

$$
-\mathrm{T}^{2} \frac{\partial}{\mathrm{T}}\left(\frac{v}{\mathrm{~T}}\right) d p-d(p v)
$$

The exact equation for $\mathrm{H}$ is easily obtained by means of a cycle similar to that employed to prove Clapeyron's equation for the connection between latent heat and vapour pressure.

Take a large (strictly, infinite) amount of a solution at pressure $p$ in osmotic equilibrium with the pure solvent at pressure $p_{0}$. It is convenient to represent the changes of pressure of solution and solvent on separate diagrams.
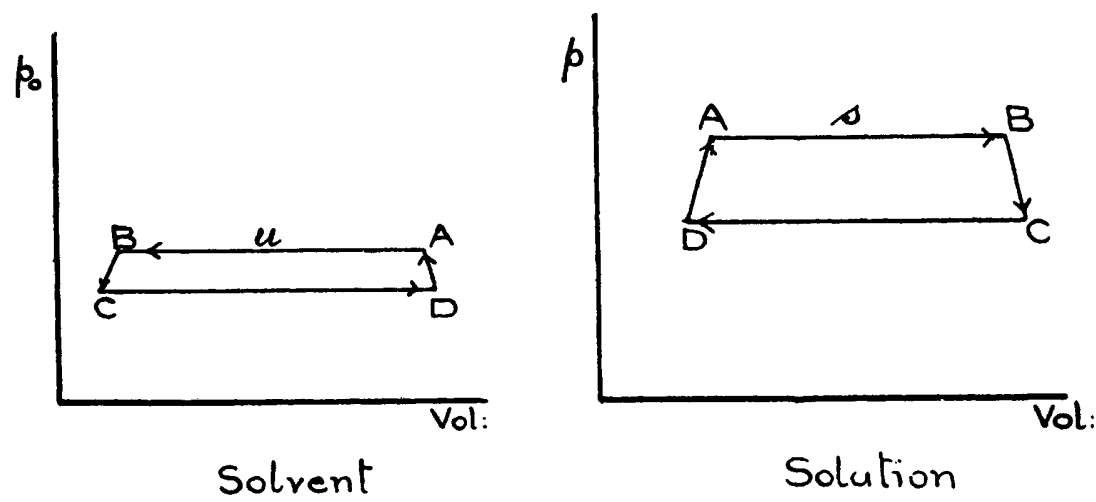

Let the solution and solvent be initially in the state A. Transfer unit mass of solvent into the solution at constant temperature $T$ through the osmotic membrane; the solution increases in volume by $s$, the solvent diminishes by $u$; both are thereby brought to the state $B$. Lower the temperature of both infinitesimally to $\mathrm{T}-d \mathrm{~T}$ without any passage of liquid across the membrane. One or both the pressures must be adjusted during the change, for the osmotic pressure $\left(p-p_{0}\right)$ varies with temperature. Let the new states be given by the points $C$. Remove the unit mass of solvent at constant temperature from the solution, through the membrane, thus bringing each to the state $D$. Warm to original temperature $T$, thus changing pressures to their old values $p$ and $p_{0}$, and bringing the system back to its original state $A$.

This cycle is a perfectly reversible one, and its mechanical efficiency may be equated to the universal value $d \mathrm{~T} / \mathrm{T}$.

\footnotetext{
- Preston, Theory of Heat, Chap. VIII, Ex. 9.
} 
The external work done is equal to the difference of the two areas $A B C D$, for they are traversed in opposite senses. This difference is--

$$
s \frac{\partial p}{\partial \mathrm{T}} d \mathrm{~T}-u \frac{\partial p_{\mathrm{o}}}{\partial \mathrm{T}} d \mathrm{~T}
$$

The heat taken in at the higher temperature $\mathbf{T}$ is $\mathrm{H}$, i.e. the reversible latent heat of dilution at that temperature; and the efficiency is therefore-

whence-

$$
\frac{s \frac{\partial p}{\partial \mathrm{T}} d \mathrm{~T}-u \frac{\partial p_{\circ}}{\partial \mathrm{T}} d \mathrm{~T}}{\mathrm{H}}=\frac{d \mathrm{~T}}{\mathrm{~T}},
$$

$$
\mathrm{H}=\mathrm{T}\left(s \frac{\partial p}{\partial \mathrm{T}}-u \frac{\partial p_{0}}{\partial \mathrm{T}}\right) . . . . . . .
$$

This very simple expression is an exact one, even for compressible liquids. Its connection with the osmotic pressure $\mathrm{P}$ is shown by making use of the definition of osmotic pressure-

and writing it-

$$
\mathrm{P}=p-p_{0}
$$

$$
\mathrm{H}=\mathrm{T}\left(\frac{\partial \mathrm{P}}{\partial \mathrm{T}}+(s-u) \frac{\partial p_{\mathrm{o}}}{\partial \mathrm{T}}\right) \text {. }
$$

The internal latent heat of dilution $\mathrm{H}_{i}$ can be obtained by subtracting the osmotic work $p s-p_{0} u$, giving the exact equation-

$$
\mathrm{H}_{i}=\mathrm{T}^{2}\left\{s \frac{\partial}{\partial \mathrm{T}}\left(\frac{p}{\mathrm{~T}}\right)-u \frac{\partial}{\partial \mathrm{T}}\left(\frac{p_{\mathrm{o}}}{\mathrm{T}}\right)\right\}
$$

in obtaining which use is made of the relation-

$$
\mathrm{T}^{2} \frac{\partial}{\partial \mathrm{T}}\left(\frac{p}{\mathrm{~T}}\right)=\mathrm{T} \frac{\partial p}{\partial \mathrm{T}}-p
$$

Now, as is well known, the osmotic pressure can be connected with the vapour pressures of solvent and solution. It was shown by the author in $1907 *$ that the exact relation is-

$$
\int_{\pi_{\pi}}^{p} s d p-\int_{\pi_{0}}^{p_{0}} u d p_{0}=\int_{\pi_{\pi}}^{\pi_{0}} v d \pi
$$

in which the second integral on the left is zero when the solvent is under the pressure of its own vapour alone.

It is at this stage that it is convenient to introduce approximations and special values.

(i) Let the solvent be under the pressure of its own vapour $p_{0}=\pi_{0}$.

(ii) Let $s-u$ be treated as negligible compared with $u$, as it always may be for ordinary aqueous solutions.

(iii) Let $s$ and $u$ be considered independent of pressure.

- Roy. Soc. Proc., A, 79, p. 519 (1907). 
We may then write-

$$
\begin{gathered}
\mathrm{H}=\mathrm{T} s \frac{\partial \mathrm{P}}{\partial \overline{\mathrm{T}}}, \\
\mathrm{H}_{i}=\mathrm{T}^{2} s \frac{\partial}{\partial \overline{\mathrm{T}}}\left(\frac{\mathrm{P}}{\mathrm{T}}\right) \\
s\left(p-\pi_{\pi}\right)=\int_{\pi_{\pi}}^{\pi_{\mathrm{o}}} v d \pi,
\end{gathered}
$$

and-

$$
p=\pi_{0}+P \text {. }
$$

If the ordinary gas law be accepted for the vapour, the integral is equal to-

$$
\text { RT } \log \frac{\pi_{0}}{\pi_{\pi}},
$$

where $R$ is the characteristic constant for the vapour; and neglecting $s\left(\pi_{\infty}-\pi_{\pi}\right)$ we have-

$$
\mathrm{P} s=\mathrm{RT} \log \frac{\pi_{0}}{\pi_{\pi}},
$$

whence-

$$
\mathrm{H}_{i}=\mathrm{T}^{2} s \underset{\partial^{\prime} \mathrm{T}}{\partial}\left(\frac{\mathrm{R}}{s} \log \frac{\pi_{0}}{\pi_{\pi}}\right)
$$

Kirchhoff's equation is at once obtained from this by taking $s$ and $R$ independent of the temperature.*

\section{Alternative Treatment.}

An alternative mode of derivation, in which the osmotic pressure does not explicitly enter, is to calculate the change in the internal energy on evaporating from the solution and subsequently condensing into pure solvent. Take a large quantity of solution under the pressure of its own vapour $\pi_{\pi}$, and evaporate I gram at constant pressure and temperature. The heat entry is-

$$
\mathrm{L}_{c}=\mathrm{T}\left(v_{\pi}-s_{\pi}\right) \frac{\partial \pi_{\pi}}{\partial \mathrm{T}^{\prime}} \text { (Clapeyron's equation), }
$$

and the change in internal energy is obtained by subtracting the external work $\left(v_{\pi}-s_{\pi}\right) \pi_{\pi}$, leaving-

$$
\mathrm{T}^{2}\left(v_{\pi}-s_{\pi}\right) \frac{\partial}{\partial \mathrm{T}}\left(\frac{\pi_{\pi}}{\mathrm{T}}\right)_{\text {sat. }}
$$

Change the pressure of the vapour at constant temperature to $\pi_{\circ}$ (the saturation value for the pure solvent) :-

Heat entry-

$$
=-\int_{\pi_{\pi}}^{\pi_{0}} \mathrm{~T}\left(\frac{\partial v}{\partial \mathrm{T}}\right) d \pi
$$

* I hope that I shall not be charged with approval either of Kirchhoff's equation or of the perfect gas law used in deriving it. As on a previous occasion I was so charged, let me say, once for all, that the above equation is now derived solely with the object of emphasizing the approximations to which it corresponds. 
work done-

$$
\int_{\pi_{\pi}}^{\pi_{0}} \pi d v=\pi_{0} v_{\pi_{0}}-\pi_{\pi} v_{\pi}-\int_{\pi_{\pi}}^{\pi_{0}} v d \pi ;
$$

change in internal encrgy-

$$
=-\int_{\pi_{\pi}}^{\pi_{0}} \mathrm{~T}^{2} \frac{\partial}{\partial \mathrm{T}}\left(\begin{array}{l}
v \\
\mathrm{~T}
\end{array}\right) d \pi-\pi_{0} v^{2} \pi_{0}+\pi_{\pi} v_{\pi} .
$$

Condense vapour into pure solvent :-

Heat entry-

$$
=-\mathrm{T}\left(v_{\pi_{0}}-u_{\pi_{0}}\right) \frac{\partial \pi_{0}}{\partial T^{\prime}}
$$

change in internal energy-

$$
=-\mathrm{T}\left(v_{\pi_{0}}-u_{\pi_{0}}\right) \frac{\partial}{\partial \mathrm{T}}\left(\frac{\pi_{0}}{\mathrm{~T}}\right)_{\text {sat. }} .
$$

The decrease in the internal energy is equal to the internal latent heat of dilution, for its value is independent of the path of change; that is-

$$
\begin{aligned}
& \mathrm{H}_{i}^{\prime}=\mathrm{T}^{2}\left(v_{\pi_{\mathrm{o}}}-u_{\pi_{0}}\right)_{\partial \mathrm{T}}^{\partial}\left(\frac{\pi_{\mathrm{o}}}{\stackrel{\mathrm{T}}{\mathrm{T}}}\right)_{\text {sat. }}-\mathrm{T}^{2}\left(v_{\pi_{\pi}}-s_{\pi_{\pi}}\right) \frac{\partial}{\partial \mathrm{T}}\left(\frac{\pi_{\pi}}{\mathrm{T}}\right)_{\text {sat }} \\
& +\int_{\pi_{\pi}}^{\pi_{0}} \mathrm{~T}^{2} \frac{\partial}{\mathrm{T}}\left(\frac{v}{\mathrm{~T}}\right)_{p} d \pi+\pi_{\mathrm{o}} v_{\pi_{\mathrm{o}}}-\pi_{\pi} v_{\pi} .
\end{aligned}
$$

This equation is exact for this particular latent heat. It differs in meaning from the previous value $H_{i}$, because in this case it is the change of energy in passing from solvent to solution, both being under the pressure of their own vapours alone; while in the former case $\mathrm{H}_{i}$ was the value when the solution was higher in pressure than the solvent by an amount equal to the osmotic pressure. As the liquids considered become more and more incompressible $\mathrm{H}_{i}$ and $\mathrm{H}_{i}^{\prime}$ tend to equality.

In a similar way the equation can be obtained which suits the more ordinary conditions, viz. that the solution and solvent are under a pressure of one atmosphere or thereabout.

We may approximate to $\mathrm{H}^{\prime}$ (i) by neglecting $u$ and $s$; (ii) by assuming vapour to follow the perfect gas law, whence-

and-

$$
\begin{aligned}
v & =\frac{\mathrm{RT}}{\pi}, \\
\pi_{0} v_{\pi_{0}} & =\pi_{\pi} v_{\pi},
\end{aligned}
$$

$\mathrm{H}_{\boldsymbol{i}}$ becomes-

$$
\partial \frac{v}{\partial T}\left(\frac{v}{\mathrm{~T}}\right)=0 \text {. }
$$

$$
\mathrm{T}^{2}\left[v_{\pi_{0}} \frac{\partial}{\partial \mathrm{T}}\left(\frac{\pi_{0}}{\mathrm{~T}}\right)-v_{\pi_{\pi} \partial} \underset{\mathrm{T}}{\partial}\left(\frac{\pi_{\pi}}{\mathrm{T}}\right)\right]
$$

or-

$$
\mathrm{H}_{i}=\mathrm{RT}^{2} \frac{\partial}{\partial T}\left(\log \begin{array}{c}
\pi_{0} \\
\pi_{\pi}
\end{array}\right)
$$

which is Kirchhoff's equation once more. 


\section{ON VON BABO'S LAW AND KIRCHHOFF'S EQUATION}

To obtain a better approximation we may take the vapour equation to be of the form-

$$
v-b=\frac{\mathrm{RT}}{p}-c \text {. }
$$

Callendar has shown that for water vapour throughout the range with which we are at present concerned $b$ may be taken as $I$ c.c., and $c$ a function of the temperature alone, which he takes as varying inversely as the $10 / 3$ power of the absolute temperature. The value of $c$ is nearly independent of pressure for moderate values of the pressure.* It may be pointed out that this equation is equivalent to retaining the rectangularhyperbolic form for the isotherms on a pv-diagram, as in the case of a perfect gas, but with the values of the volumes corresponding to any value of $p$ on a given isotherm decreased by the amount $c-b$. At less moderate pressures $c$ cannot be taken as independent of the pressure, so it is not surprising that the equation gives no indication of critical phenomena; as is evident from the fact that there are no kinks in a rectangular hyperbola. The values of $c$ which he gives are-

\begin{tabular}{|c|c|c|c|c|}
\hline \multicolumn{3}{|c|}{ Temp. ${ }^{\circ} \mathrm{C}}$. & \multicolumn{2}{|c|}{$c$ (in c.c.) } \\
\hline o & $\ldots$ & ... & $\ldots$ & $74^{\circ} 43$ \\
\hline 20 & $\ldots$ & ... & $\ldots$ & $5^{8 \cdot 81}$ \\
\hline 40 & ... & ... & $\ldots$ & $47^{\circ} 19$ \\
\hline 60 & $\ldots$ & $\ldots$ & $\ldots$ & $3^{8 \cdot 68}$ \\
\hline 80 & ... & ... & $\ldots$ & $31^{\circ} 60$ \\
\hline 100 & $\ldots$ & ... & $\ldots$ & $26 \cdot 30$ \\
\hline
\end{tabular}

The value of $R$ he takes as $111030 \frac{\text { cal. }}{\text { gr. deg. C: }}$

Using this equation we will also put $u=s=b$. Then-

$$
\begin{aligned}
\mathrm{H}_{i}^{\prime} & =\left(\frac{\mathrm{RT}}{\pi_{0}}-c\right) \frac{\partial}{\partial \mathrm{T}}\left(\frac{\pi_{0}}{\mathrm{~T}}\right)-\left(\frac{\mathrm{RT}}{\pi}-c\right) \frac{\partial}{\partial \mathrm{T}}\left(\frac{\pi}{\mathrm{T}}\right) \\
& -\frac{\partial}{\partial \mathrm{T}} \frac{c-b}{\mathrm{~T}} \cdot\left(\pi_{0}-\pi\right)+\frac{\pi_{0}}{\mathrm{~T}^{2}}\left(\frac{\mathrm{RT}}{\pi_{0}}-c+b\right)-\frac{\pi}{\mathrm{T}^{2}}\left(\frac{\mathrm{RT}}{\pi}-c+b\right),
\end{aligned}
$$

which on collecting the various terms reduces to-

$$
\frac{H_{i}^{\prime}}{T^{2}}=R_{\frac{\partial}{\partial T}}\left(\log \frac{\pi_{0}}{\pi}\right)-\frac{\partial}{\partial T}\left(\frac{c\left(\pi_{0}-\pi\right)}{\mathrm{T}}\right) .
$$

If we apply Callendar's equation also to the value of $\mathrm{H}$ calculated by the former method, we have first-

$$
\left(\mathrm{P}+\pi_{\mathrm{o}}-\pi\right) s=\int_{\pi}^{\pi_{\mathrm{o}}} v d \pi=\int_{\pi}^{\pi_{\mathrm{o}}}\left(\frac{\mathrm{RT}}{\pi}-c+b\right) d \pi=\mathrm{RT} \log \frac{\pi_{\mathrm{o}}}{\pi}-c\left(\pi_{\mathrm{o}}-\pi\right),
$$

where $(b-s)\left(\pi_{0}-\pi\right)$ is finally neglected. Thence-

and-

$$
\mathrm{H}=\mathrm{T} s_{\partial \mathrm{T}}^{\partial \mathrm{P}}=\mathrm{T}_{\frac{\partial}{\partial \mathrm{T}}}\left\{\mathrm{RT} \log \frac{\pi_{0}}{\pi}-c\left(\pi_{0}-\pi\right)\right\},
$$

$$
\mathrm{H}_{i}=\mathrm{T}^{\mathrm{s}} \mathrm{\partial} \frac{\partial}{\partial \mathrm{T}}\left(\frac{\mathrm{P}}{\mathrm{T}}\right)=\mathrm{T} \frac{\partial}{\partial \mathrm{T}}\left[\mathrm{R} \log \frac{\pi_{0}}{\pi}-c \frac{\pi_{0}-\pi}{\mathrm{T}}\right] .
$$

- Or rather for moderate densities. 
Here $s$ is considered independent of temperature as before. This equation, to the degree of approximation obtained, is identical with the value of $\mathrm{H}^{\prime}$ derived above.

If the value of $\mathrm{H}_{i}$ is zero at all temperatures-

is constant.

$$
R \log \frac{\pi_{0}}{\pi}-c \frac{\left(\pi_{0}-\pi\right)}{T}
$$

Von Babo's law is obtained by neglecting the variation of the second term with temperature. For the law to be true, then, to the accuracy of the above equation-

$$
\frac{\mathrm{H}_{i}^{\prime}}{\mathrm{T}^{2}}=-\left(\mathrm{I}-\frac{\pi}{\pi_{0}}\right) \frac{\partial}{\partial \mathrm{T}}\left(\frac{c \pi_{0}}{\mathrm{~T}}\right)
$$

The value of $\frac{c \pi_{\circ}}{\mathrm{T}}$ for water vapour at $60^{\circ} \mathrm{C}$. is $\mathrm{I}^{\circ} \mathrm{7}$ and at $80^{\circ} \mathrm{C}$. is $3^{.2}$. Hence $\frac{\mathrm{H}_{i}^{\prime}}{\mathrm{T}^{2}}$ would need to be-

$$
\begin{aligned}
& -\left(\mathrm{I}-\frac{\pi}{\pi_{0}}\right) \times \frac{155}{20} \times 13,300 \\
= & -\left(\mathrm{I}-\frac{\pi}{\pi_{0}}\right) \times 1,000 \mathrm{ergs} /(\text { deg. C. })^{2}
\end{aligned}
$$

or, since $\frac{\pi}{\pi_{0}}$ is less than unity, $H_{i}^{\prime}$ would need to be negative.

If, on the other hand, $\mathrm{H}_{i}^{\prime}$ is independent of temperature-

$$
\mathrm{R} \log \frac{\pi_{\mathrm{o}}}{\pi}-c \frac{\left(\pi_{\mathrm{o}}-\pi\right)}{\mathrm{T}}=-\frac{\mathrm{H}_{i}}{\mathrm{~T}}+\text { const. }
$$

On the Physical Meaning of Kirchhoff's Equation.

So far I have treated the question purely from the thermodynamical standpoint. Useful though such an examination is, yet the physicist cannot stop here. The further question as to the mechanism which may account for the observed facts insistently obtrudes itself.

The kinetic theory of matter, which until recently was a speculation only, has become an experimental fact owing to the researches of Perrin and others on "molecular reality" and Brownian motion. We need no longer, then, have any hesitation for the case of very dilute solutions (in which the solute molecules are so far apart on the average as to exert no sensible attraction on one another) in applying the same equation as for a similar gas, viz.-

$$
\mathrm{P}(v-b)=\mathrm{RT},
$$

where $v=$ volume containing I gram of solute and $\mathrm{P}$ is the osmotic pressure. Take now our nearly exact equation for $\mathrm{H}_{i}$, viz.-

$$
\mathrm{H}_{i}=\mathrm{T}^{2} s \frac{\partial}{\partial \mathrm{T}}\left(\frac{\mathrm{P}}{\overline{\mathrm{T}}}\right)
$$

and we obtain $\mathrm{H}_{i}=0$, since-

$$
\frac{\mathrm{P}}{\mathrm{T}}=\frac{\mathrm{R}}{v-b} .
$$

Thus in this case there would be no heat of dilution; at least, this is so provided that the molecular weight of the solute keeps constant (i.e. $R=$ const.).

vOL. XI-T2 
This will not keep so if there is association or dissociation of solute molecules, either with themselves or with solvent molecules (provided in the latter case that the union is of the same permanent or subpermanent character as in the former-such, in fact, that the combination moves about as a physical unit). Such a change in association alters the molecular weight and therefore the value of $R$, which varies inversely as it. In consequence $\underset{T}{P}$ would no longer be independent of temperature. It is unlikely, however, that when the solution is so dilute as to make the solute molecules independent of each other, as supposed, that any further separation of these molecules should cause any change in their character.

When the molecules are so crowded that one can no longer neglect the forces between them, we must copy what Hirn did for gases," and write-

$$
(\mathrm{P}+\mathrm{K})(v-b)=\mathrm{RT} \text {, }
$$

where $\mathrm{K}$ is the so-called internal pressure due to molecular attractions. If $\mathrm{K}$, as wel! as $R$, is independent of temperature, we obtain-

$$
\mathrm{H}_{i}=\mathrm{T}^{2} s \frac{\partial}{\partial \mathrm{T}}\left(\frac{\mathrm{P}}{\mathrm{T}}\right)=\mathrm{Ks} \text {. }
$$

Hence the internal latent heat of dilution would represent simply the work done against molecular forces during the dilution. $f$ This might, so far as we know a priori, be either positive or negative, because we cannot state offhand whether the solute molecules attract or repel one another when in the body of the solution. It must not be forgotten that the action is a differential one ; for as soon as the volume of the molecule is taken into account it can only be present by displacing one (or more or less) solvent molecule. If the attraction between the displaced solvent and a solute molecule is greater than between the two solute molecules, the effective action will be a repulsion; in other cases it will be an attraction or nil. The fact that in the case of sugar in water the latent heat of dilution is fairly small renders plausible the supposition that this differential action is only small. On the other hand, it may be that in measuring the heat of dilution we are only observing a balance of effects arising in part from separation of the distances between solute molecules, and in part from change of association of these with each other'or with solvent molecules. In this connection I may state that Lord Berkeley's values of osmotic pressure at constant temperature are fairly well represented by a formula $\mathrm{P}(v-b)=$ constant, which would seem to imply (since $\mathrm{K}$ is absent) that the differential attractions are quite small. It is not safe positively to assert its smallness, because in such a case the effects of $b$ and $K$ may possibly be run together into a single term, as is done by Callendar in his gas equation for steam at moderate pressures.

We will next discuss the reason of the abnormal lowering of vapour pressure in the case of strong solutions. We have seen that-

or therefore-

$$
\text { Ps }=\text { RT } \log \frac{\pi_{0}}{\pi} \text { nearly, }
$$

$$
\mathrm{P}=\frac{\mathrm{R}_{\mathrm{o}} \mathrm{T}}{\mathrm{M}_{\text {vap }} \mathrm{s}} \log \frac{\pi_{0}}{\pi}
$$

where $R_{o}$ is the value of $R$ per gram molecule.

* Hirn, 1865. Often attributed to van der Waals, whose contribution (188r) consisted in finding a particular value for $K$ and deducing the consequences.

$t$ If $K$ is a function of the temperature this equality no longer holds good.

t Hence the difficulty in discriminating between van Laar and Dolezalek and their respective schools. 


\section{FOR THE LATENT HEAT OF DILUTION}

Let the solution consist of $n$ molecules of solute dissolved in $\mathrm{N}$ molecules of solvent, each reckoned as of the same complexity as in the vapour state ; let $\mathrm{V}_{w}$ be put equal to $\mathrm{NM}_{\text {vap. }} \mathrm{S}$; and let $\mathrm{V}$ be the total volume of the same solution, containing therefore $n$ molecules of solute. Then-

$$
\mathrm{P}=\frac{n \mathrm{R}_{\mathrm{o}} \mathrm{T}}{\mathrm{V}-n b}=\frac{\mathrm{NR}_{\mathrm{o}} \mathrm{T}}{\mathrm{V}_{w}} \log \frac{\pi_{\mathrm{o}}}{\pi}, \cdots
$$

whence the equation for the lowering becomes-

$$
\log \frac{\pi_{\mathrm{o}}}{\pi}=\frac{n}{\mathrm{~N}} \frac{\mathrm{V}_{w}}{\mathrm{~V}-n b} .
$$

We might at first be tempted to write-

$$
\mathrm{V}_{w w}=\mathrm{V}-n b,
$$

in which case Raoult's law would be true for strong solutions (except for the introduction of the logarithm). Experiment shows that this is by no means the case-in fact, $\mathrm{V}_{w}$ is greater than $\mathrm{V}-n b$. Let us suppose, as Callendar and others have done, that the molecules of solute become hydrated, each combining with $a$ molecules of solvent. Then if there is no contraction, except what is represented by the difference between $s$ and $u$ for the water, we have-

$$
\mathrm{V}=n b+(\mathrm{N}-n a) s \mathrm{M}_{\text {vap. }}
$$

where $b$ represents the volume of a hydrated molecule, and therefore includes the volume of $a$ molecules of solvent, and therefore-

$$
s \mathrm{M}_{\text {vap. }}=\frac{\mathrm{V}-n b}{\mathrm{~N}-n a},
$$

so that $\log \frac{\pi_{\mathrm{o}}}{\pi}$ becomes $=\frac{n}{\mathrm{~N}-\mathrm{an}}$.

This is exactly the formula which Callendar employs. But although the interpretation of $a$ as the hydration factor is the one which he accepts, yet the reason of its entry into this equation would appear to be different from that which he himself gives. In our formula it appears as an effect of alteration of the volume of the solute molecules-each molecule of the hydrated salt being larger than that of the unhydrated salt, owing to the water molecules combined with it. On the other hand Callendar, developing ideas which had been foreshadowed by Poynting, interprets the effect of the hydration as being to render ineffective for evaporation a number $a$ of molecules of solvent for each molecule of solute. There is a difficulty in this

* This might also have been obtained from the mathematical identity-

$$
\left(\frac{\partial \pi}{\partial n}\right)_{\hat{p}}\left(\frac{\partial n}{\partial p}\right)_{\pi}\left(\frac{\partial p}{\partial \pi}\right)_{n}=-\mathrm{I}
$$

taken together with the values-

this gives-

$$
\left(\frac{\partial \pi}{\partial p}\right)_{n}=\frac{s}{i^{\prime}}=\frac{s M_{\mathrm{vav},} \pi}{\mathrm{R}_{\mathrm{o}} \mathrm{T}} \quad\left(\frac{\partial p}{\partial n}\right)_{\pi}=\frac{n \mathrm{R}_{\mathrm{o}} \mathrm{T}}{\mathrm{V}-n b}
$$

whence by integration--

$$
-\left(\frac{\partial \pi}{\partial n}\right)_{p}=\frac{s \mathbf{M}_{\mathrm{vap}} \pi}{\mathrm{R}_{\mathrm{o}} \mathrm{T}} \cdot\left(\frac{\partial p}{\partial n}\right)_{\pi}
$$

$$
\log \frac{\pi_{\mathrm{o}}}{\pi}=\frac{s \mathrm{M}_{\mathrm{o}} n}{\mathrm{~V}-n b}=\stackrel{n}{\mathrm{~N}} \frac{\mathrm{V}_{w}}{\mathrm{~V}-n b} .
$$

In this proof we have taken $p$ and $\mathrm{P}$ as practically equal. 
latter interpretation which has not yet been pointed out. According to it, $\mathrm{N}$ - $\mathrm{an}$ is the number of free molecules of solvent present in the liquid. But we have seen that $\mathrm{N}$ is not the total number in the liquid (even including those combined with the solvent) but the number that would be in the same mass of vapour formed from the liquid. Now there is fairly strong evidence that water contains a large number of associated molecules (i.e. molecules combined with each other, $2 \mathrm{H}_{2} \mathrm{O}, 3 \mathrm{H}_{2} \mathrm{O}$, etc.), * so that the real number of liquid molecules in the absence of the solute would not be $N$ but (say) $\beta N$. If then we imagine each of the $n$ molecules of solute to render inactive any number $a$ of these, the number of active molecules would be $\beta \mathrm{N}-\alpha$, not $\mathrm{N}-a n$. The theory evidently breaks down in fact, for the form in which it is usually stated even for infinitely dilute solutions, unless we give up the supposition that water (liquid) is associated. It is possible that by some inconsiderable change in the statement of this theory the difficulty might be removed; but the change has not yet been suggested.

The explanation given above of the term an makes it proportional to the increase in the effective volume of the molecules of the solute due to hydration. When this effective volume is calculated (from osmotic pressures or lowering of vapour pressures or any other phenomena into which it enters in the same way) it comes out bigger than the volume expected from the density and molecular weight of solid sugar ; and this may be explained by the hydration of the solute, the size of the molecules being thereby increased. The value for the hydration comes out the same as on Callendar's theory, but the underlying conception is totally different.

\section{AdDEndum.}

The following alternative way of finding $\mathrm{H}_{i}$ is very simple.

For any homogeneous fluid we may write : Increase of internal energy-

$$
\begin{aligned}
=d \mathrm{E} & =\mathrm{S} d \mathrm{~T}+\mathrm{T}\left(\frac{\partial p}{\partial \mathrm{T}}\right)_{v} d \mathrm{~V}-p d \mathrm{~V}, \\
& =\mathrm{S} d \mathrm{~T}+\mathrm{T}^{2} \frac{\partial}{\partial \mathrm{T}}\left(\frac{p}{\mathrm{~T}}\right) d \mathrm{~V} .
\end{aligned}
$$

When we have two phases at different pressures $p$ and $p_{0}$ and volumes $\mathrm{V}$ and $\mathrm{V}_{0}$ we may similarly write-

$$
d \mathrm{E}=\mathrm{S} d \mathrm{~T}+\mathrm{T}^{2} \frac{\partial}{\partial \mathrm{T}}\left(\frac{p}{\mathrm{~T}}\right) d \mathrm{~V}+\mathrm{T}^{2} \frac{\partial}{\partial \mathrm{T}}\left(\frac{p_{0}}{\mathrm{~T}}\right) d \mathrm{~V}_{0}
$$

where $E$ and $S$ are the energy and the thermal capacity of the double system.

Now in an isothermal change in which $d \mathrm{~V}=s$ and $d \mathrm{~V}_{0}=-u$, the value of $d \mathrm{E}$ is what we have called $\mathrm{H}_{i}$, i.e.-

$$
\mathrm{H}_{i}=\mathrm{T}^{2}\left[s \frac{\partial}{\partial \mathrm{T}}\left(\frac{p}{\mathrm{~T}}\right)-u \frac{\partial}{\partial \mathrm{T}}\left(\frac{p_{\mathrm{o}}}{\mathrm{T}}\right)\right]
$$

in which we remove the restriction as to constant volume during the differentiation as $p$ and $p_{0}$ are independent of the volumes for the changes of volume involved.

* See, for example, "General Discussion on the Constitution of Water," Trans. Farad. Soc., July, I910, where numerous references are given. 